\title{
Personalized medicine in prostate cancer: future perspectives for tailored treatments
}

\begin{abstract}
Best future standard of care will be the identification of "tailored" treatments, based on the specific cancer patient's characteristics, race differences and habits or regional backgrounds, or clinical aspects, etc. The development of tools that allow and support the decisionmaking process to deliver tailored treatment is crucial as medicine and in particular cancer disciplines move towards the era of individualized medicine. This aim is proposed by designing, developing and testing a framework to represent data in a re-usable way for building automatically Decision Support Systems based on extrapolated predictive models.

Extracting the main features from classical and innovative data, DSS will be able to provide a practical support to clinical choices in cancer matter. A multidisciplinary integration with different professionalisms beside the clinicians is a necessary step to follow this important issue.
\end{abstract}

Keywords: personalized medicine, predictive models, decision support systems

Volume 3 Issue 5 - 2015

\section{Alitto AR, Gatta R, Meldolesi E, Masciocchi C, Damiani A, Lanzotti V,Valentini V, Mantini $\mathrm{G}$}

Department of Radiation Oncology, Catholic University of the Sacred Heart, Italy

Correspondence: Alitto AR, Radiation Oncology Department, Gemelli-ART, Catholic University of the Sacred Heart, Rome, Italy, Tel 3.9IE+ II, Fax 630155908, Email annarita.alitto@rm.unicatt.it

Received: October 25, 2015 | Published: November 27, 2015
Abbreviations: PM, personalized medicine; DSS, decision support systems; ML, machine learning; SIO, semantic interoperability; NCI, National cancer institute thesaurus; OBO, open biomedical ontologies; FMA, foundational model of anotomy; ANNs, artificial neural networks; BNs, bayesian networks; SVMS, support vector machines; DTs, decision trees

\section{Introduction}

Personalized Medicine (PM) has been one of the principle aims of cancer care in the last years. Advances in Radiation Oncology and, in general, in modern medicine made more complex the "personalized medicine" process " "Personalized medicine" is defined by the National Cancer Institute as a "form of medicine that uses information about a person's genes, proteins, and environment to prevent, diagnose, and treat disease. In cancer, personalized medicine uses specific information about a person's tumour to help diagnose, plan treatment, find out how well treatment is working, or make a prognosis". ${ }^{2} \mathrm{New}$ interesting methodologies and tools are now available to realize this important issue.

\section{Perspectives}

To reach a clinical choice "tailored" on a single patient, clinicians have to consider even more biometric parameters and clinical features, i.e. genomics, blood tests, imaging, and this growing complexity cannot be tackled only by human brain. ${ }^{3}$ In addition, with the continuous development of medicine, many therapeutic options are now available.

In this scenario, clinical choices are, traditionally, based on guidelines, derived from large randomized trials and meta-analyses; however, population enrolled represent only a small and homogeneous selected sample. However, large randomized trials and meta-analyses or systematic review have a key role, they need the integration of new different approaches, such as the results of observational studies, ant the consideration of patients' features difference. ${ }^{4}$ Nowadays, population-based observational studies emerge as complementary to randomized clinical trials, to translate the benefits of trials themselves in the general population. ${ }^{5}$
For these large amounts of features regarding the patients and also the tumours, in combination with the increase of therapeutic options, it is hard to "trial" every possible assortment. ${ }^{6}$ The increasing number of treatment options now available, the heterogeneity features among patients and the same tumours themselves (e.g. molecular and genomic differences), the consequent difficult translation of the trials results to the general population and the numerous published studies, render the identification of the best treatment, for a single cancer patient, very difficult.

In particular in radiotherapy, because of the increasing of better planning and delivery facility evolutions, future practice will need to take into account the increasing available treatment options, innovations and patient data. In fact, more advanced technologies have been adopted in clinical use in the last decade, such as image guided radiation therapy, different advanced dose modulation and delivery techniques, particle therapy, with a increasing attention to address resources in a reliable and cost-effective technologies. ${ }^{4}$

Furthermore, radiotherapy is actually more a biological intervention rather than a physical, because of its effects on cellular and molecular level ${ }^{7,8}$ the heterogeneity of dose distribution in the irradiated and normal tissue should be considered. ${ }^{1}$ In the past, in order to analyse the treatment effects in radiation oncology, several models were elaborated, to show the correlation between radiotherapy dose and outcomes, through different dose-reduction methods; the probit-based one (or the Lyman-Kutcher- Burman model) ${ }^{9}$ and the logit-based one ${ }^{10}$ are the most used dose-response models. Many times, the heterogeneity of dose distribution has been represented by a single value correlated with the outcome itself, the Equivalent Uniform Dose, developed by Niemerko. ${ }^{11}$ But numerous factors influenced the outcomes, and these factors can hardly be summarized in the models cited before, because the fitting processes underlying the dose-response modelling creation usually involves no more than two or three parameters describing the geometry of sigmoid dose-response curves, namely tumour control probability and normal tissue complication probability. ${ }^{4}$ Even if some correction could be introduced to improve these models, in many case the number of variables and of patients, to be included or evaluated to obtain a new model, can be very large. ${ }^{4}$ 
This large amount of data, that grows up rapidly and that comes from various sources and collects heterogeneous characteristics, is essential to converge towards an individualized medicine. In this context, extensive, multifactorial data sharing is a crucial prerequisite for current and future (radiotherapy) research. ${ }^{12}$

All these considerations are true for all cancer types, even for prostate cancer, the most common tumour in the male occidental population (250.000 new diagnoses in USA during 2012) ${ }^{13}$ with an expecting growing up prevalence in the following years $(220.800$ estimated new case in 2015). ${ }^{14}$ In this cancer type, more than in others, the therapeutic options are numerous, including surgery, with its different modalities, radiotherapy, with many techniques, dose and dose per fraction, hormone therapy alone or in association with other treatments, active surveillance, or others evolving therapies like cryosurgery or high intensity focused ultrasound (HIFU). ${ }^{15}$ So, with the heterogeneity of tumours and patients, the current speed of innovations and of new available options, the increasing numbers of published papers, it is very difficult to make a right choice for the single prostate cancer patient that we usually meet in our clinical practice.

In the last decades, in order to contribute to personalized medicine aim, also computer science has playing a key role, ${ }^{16}$ promoting a rapid learning approach by analysing a large amount of data to support clinical decisions. ${ }^{17}$

In this context, a possible solution, towards a personalized medicine issue, is the development of predictive models to create Decision Support Systems (DSSs). Decision Support Systems could help radiation oncologists and clinical oncologists in these issues in many phases of the patient's clinical pathway (estimation of treatment effects, induced toxicities, costs/benefits evaluation, resource allocation, etc.).

Decision Support Systems are Systems built by the most recent techniques coming from the world of Artificial Intelligence: both for maths algorithms to build regressors and classifiers and for languages to represent the involved clnical knowledge. Even if the computers play a crucial role in computation of such algorithms the deployment of the results can be done in many different ways, for example by nomograms, smatphone, etc. Such DSSs, in addition, is becoming even more a multidisciplinary task that involves not only radiation oncologists or other physicians from different specialty, but also mathematicians, engineers, physics and computer scientists. Collecting and analysing a large amount of heterogeneous data from different data sources, in terms of space and time, are the challenge of these multidisciplinary teams.

DSSs require, for the multiple variables to consider, large heterogeneous databases, not only to create a prediction model with a good statistical power, but also to validate it. Hence to create large databases, it grows the necessity to share and combine multiple datasets, often horizontally (data collected across institutes) and vertically (single patient's features stored in separate datasets) partitioned. ${ }^{18}$ Innovative "rapid learning" research techniques allow extraction of knowledge of the masses for the benefit of the individual. ${ }^{17}$

The developing of prediction models based on large databases approach requires a flexible strategy for data collection, data mining and outcome reporting, quite different from the pre- determined aim of a prospective randomized controlled trial. Collecting data, without knowing beforehand what the relevant features will be, implies a standardization of any variable to collect to universally define data and procedures allowing automatic and consistent upload from any data sources. ${ }^{1}$

This process will take advantage of an ontology definition to describe data, both for being sure to share the same meanings between researchers and to allow automatic inference/reasoning from computers. Ontology "is a (formal) specification of concepts, relations and functions in a domain and hence focuses on concepts". ${ }^{19}$ Medical ontology is a model of the knowledge from a clinical domain; it contains all the relevant concepts, related to a clinical field, organized in a formal way that allows knowledge inference and reasoning. The advantages of using ontology for knowledge representation are: standardization of medical terms, knowledge sharing and also support for automatic reasoning. ${ }^{20}$

In particular, Semantic interoperability (SIO) is crucial, to share data, reducing at the minimum the entropy of the information flow. Many ontologies have been already developed, just because data integration is a crucial tool for biomedical researches. ${ }^{21}$ Examples are Open Biomedical Ontologies (OBO), ${ }^{22}$ Foundational Model of Anotomy (FMA), ${ }^{23}$ National Cancer Institute Thesaurus (NCI), ${ }^{24,25}$ Medicine-Clinical Terms (SNOMED-CT), ${ }^{26}$ and many others.

After collection, the data has to be analysed in order to create multifactorial predictive models, to build one or more DSSs. Several interactive DSS have been created in the last years, currently used in clinical practice, for radiotherapy (www.predictcancer.org, www. mskcc.org/nomograms, etc) and also for prostate cancer issues (Partin Tables, Kattan nomograms, D'Amico tables, etc), ${ }^{27-29}$ quite all task oriented; DSSs predictive models could be represented in different ways: by nomograms, by interactive websites, or also by specific applications for devices.

Such DSSs need validation to clearly identify and describe performances and limitations. Moreover, modern approaches, coming from the discipline of Computer Science and Artificial Intelligence, allow facing this challenge with a rich set of tools overcoming the limitations of the classical statistical approach. ${ }^{30}$

Besides mathematical and statistical modelling (regression, survival models), Machine Learning methods is a data analysis method that could render automatic the process of models building. Machine Learning (ML) utility over classical statistical methods has already been demonstrated and is a promising tool to develop predictive models. ${ }^{31}$

ML is a branch of artificial intelligence that employs a variety of statistical, probabilistic and optimization techniques that allows computers to "learn" from past examples and to detect hard-to-discern patterns from large, noisy or complex data sets. This capability is particularly well suited to medical applications, especially those that depend on complex proteomic and genomic measurements. As a result, machine learning is frequently used in cancer diagnosis and detection and recently applied to cancer prognosis and prediction. ${ }^{31}$

This latter application is particularly interesting for personalized, predictive medicine tasks. In addition, the ability of ML tools to detect key features from complex datasets reveals their importance. A variety of these techniques, including Artificial Neural Networks (ANNs), Bayesian Networks (BNs), Support Vector Machines (SVMs) and Decision Trees (DTs) have been widely applied in cancer research for the development of predictive models, resulting in effective and accurate decision-making. Even though it is evident that the use of ML methods can improve our understanding of cancer progression, 
to include these methods in daily clinical practice a strong validation is needed. ${ }^{32}$

To reach data reusability through time, space (different Institutions), or in different research contexts, DSSs plays a key role, overcoming traditional methods for decision-making. Nowadays, most DSSs are based on the outcome of a specific randomized clinical study: in this process, data collection is essentially "goal-oriented".

If these two approaches seem to be in contradiction, in reality they are complementary. The key value of randomized clinical trials and consequent meta-analyses and reviews cannot be ignored, but some of their limits are the long follow up needed to obtain the established outcomes, the accrual of only homogeneous subgroups of population with determinate characteristics and also the lack of standardized assessments among the different studies, in terms of methodology, patients' characteristics, outcomes, data collection systems, etc. Emerging observational studies are crucial to confirm the trials results in the general population, more heterogeneous and with different characteristics, and also to identify whether practice has changed appropriately (Booth 2014); moreover, they could facilitate the identification of risks groups and, consequently, the choice of a better treatment for each patient; they could, also, stimulate research focused on specific risk groups.

\section{Conclusion}

Predictive models could be complementary to guidelines; towards a more tailored decision making process ${ }^{18}$ and DSSs will be able to provide a practical support.

The future of the cancer research is based on the integration and collaboration of multidisciplinary fields, for a hybrid discipline encompassing the fields of oncology, computer science, bioinformatics, statistics, computational biology, genomics, proteomics, metabolomics, pharmacology, and quantitative epidemiology. The common challenge is the need to bring order to the massive amounts of data generated by researchers and clinicians attempting to find the underlying causes and effective means of diagnosing and treating cancer. $^{33}$

The knowledge in these fields and the multidisciplinary collaboration among different figures, are the key to develop accurate and scientifically based decision tools, for a more personalized decision-making process.

\section{Acknowledgments}

The authors have no relevant affiliations or financial involvement with any organization or entity with a financial interest in or financial conflict with the subject matter or materials discussed in the manuscript This includes employment, consultancies, honoraria, stock ownership or options, expert testimony, grants or patents received or pending or royalties

\section{Conflicts of interest}

The authors declare there is no conflict of interests.

\section{Funding}

None.

\section{References}

1. Meldolesi E, van Soest J, Alitto AR, et al. VATE:VAlidation of high TEchnology based on large database analysis by learning machine. Colorect Cancer. 2015;3(5):435-450.
2. NCI Dictionary of terms:personalized medicine. NCI

3. Abernethy AP, Etheredge LM, Ganz PA, et al. Rapid-learning system for cancer care. J clin Oncol. 2010;28(27):4268-4274.

4. Valentini V, Dinapoli N, Damiani A. The future of predictive models in radiation oncology:from extensive data mining to reliable modeling of the results. Future Oncol. 2013;9(3):311-313.

5. Booth CM, Tannock IF. Randomised controlled trials and populationbased observational research:partners in the evolution of medical evidence. Br J Cancer. 2014;110(3):551-555.

6. Dekker ALAJ, Gulliford SL, Ebert MA, et al. Future radiotherapy will be based on evidence from retrospective interrogation of linked clinical data sources rather than prospective randomized controlled clinical trials. Med Phys. 2014;41(3):030601.

7. Bentzen SM. Preventing or reducing late side effects of radiation therapy:radiobiology meets molecular pathology. Nat Rev Cancer. 2006;6(9):702-713.

8. Fowler JF. 21 years of biologically effective dose. $\mathrm{Br} J$ Radiol. 2010;83(991):554-568.

9. Burman C, Kutcher GJ, Emami B, et al. Fitting of normal tissue tolerance data to an analytic function. Int J Radiat Oncol Biol Phys. 1991;21(1):123-135.

10. Gay HA, Niemerko A. A free program for calculating EUD-based NTCP and TCP in external beam radiotherapy. Phys Med. 2007;23(34):115-125.

11. Niemerko A. Reporting and analyzing dose distributios:a concept of equivalent uniform dose. Med Phys. 1997;24(1):103-110.

12. Roelofs E, Dekker A, Meldolesi E, et al. International data-sharing for radiotherapy research:an open-source based infrastructure for multicentric clinical data mining. Radiother Oncol. 2014;110(2):370 374

13. Aizer AA, Paly JJ, Efstathiou JA. Multidisciplinary care and management selection in prostate cancer. Semin Radiat Oncol. 2013;23(3):157-164.

14. Siegel RL, Miller KD, Jemal A. Cancer statistics, 2015. CA Cancer J Clin. 2015;65(1):25-29.

15. Mantini G, Alitto AR, Fionda B, et al. Radiotherapy in men with prostate cancer:indications, evolutions and integrated approaches. Urologia 2013;80(3):188-201.

16. Meldolesi E, van Soest J, Dinapoli N, et al. Medicine is a science of uncertainty and an art of probability (Sir W. Osler). Radiother Oncol. 2015;14(1):132-134.

17. Lambin P, Roelofs E, Reymen B, et al. Rapid learning health care in oncolog -an approach towards decision support systems enabling customised radiotherapy. Radiother Oncol. 2013;109(1):159-164.

18. Meldolesi E, van Soest J, Dinapoli N, et al. An umbrella protocol for standardized data collection (SDC) in rectal cancer:a prospective uniform naming and procedure convention to support personalized medicine. Radiother Oncol. 2014;112(1):59-62.

19. Gruber TR. Toward Principles for the Design of Ontologies Used for Knowledge Sharing. International Journal of Human-Computer Studies. 1995;43(5-6):907-928.

20. Gamberger D, Prcela M, Jovic A. Semantic web ontology utilization for heart failure expert system design. Stud Health Technol Inform. 2008; 136:851-856.

21. Min H, Manion FJ, Goralczyk E, et al. Integration of prostate cancer clinical data using an ontology. J Biomed Inform. 2009;42(6):10351045 .

22. OBO. The OBO Foundry. 2009. 
23. Rosse C, Mejino JL. A reference ontology for biomedical informatics:the Foundational Model of Anatomy. J Biomed Inform. 2003;36(6):478500 .

24. Golbeck J, Fragoso G, Hartel F, et al. The national cancer institute's thesaurus and ontology. J Web Seman. 2003;1(1):75-80.

25. Sioutos N, de Coronado S, Haber MW, et al. NCI thesaurus:a semantic model integrating cancer-related clinical and molecular information. $J$ Biomed Inform. 2007;40(1):30-43.

26. SNOMED-CT The global language of healthcare.

27. Partin tables.

28. Prostate Cancer. Memorial sloan kettering cancer center.

29. D'Amico AV, Whittington R, Malkowicz SB, et al. Pretreatment nomogram for prostate-specific antigen recurrence after radical prostatectomy or external-beam radiation therapy for clinically localized prostate cancer. J Clin Oncol. 1999;17(1):168-172.
30. Vallati M, De Bari B, Gatta R, et al. Exploiting Machine Learning for Predicting Nodal Status in Prostate Cancer Patients. Artificial Intelligence Applications and Innovations IFIP Advances in Information and Communication Technology. 2013;412:61-70.

31. Cruz JA, Wishart DS. Applications of machine learning in cancer prediction and prognosis. Cancer Inform. 2007;2:59-77.

32. Kourou K, Exarchos TP, Exarchos KP, et al. Machine learning applications in cancer prognosis and prediction. Comput Struct Biotechnol J. 2014;13:8-17.

33. Jiang H, An L, Baladandayuthapani V, et al. Classification, predictive modelling, and statistical analysis of cancer data (a). Cancer Inform. 2014;13(Suppl 2):1-3. 